\title{
Supplementary Information: Small molecule
} modulation of intrinsically disordered proteins using molecular dynamics simulations

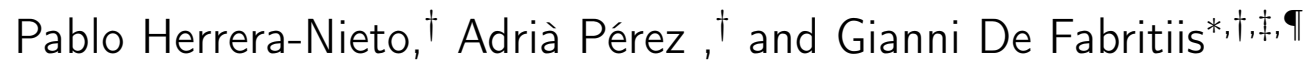 \\ $\dagger$ Computational Science Laboratory, Universitat Pompeu Fabra, Barcelona, Spain \\ $\ddagger$ Acellera Ltd., Barcelona, Spain \\ 【Institució Catalana de Recerca i Estudis Avançats, Barcelona, Spain \\ E-mail: gianni.defabritiis@upf.edu
}




\section{$K_{e q}$ calculation}

For the computation of $K_{e q}$ it is first required to compute the free energy. Free energy for the bound state was calculated on the basis of the equilibrium distribution using the equation.

$$
\Delta G=-k_{B} T \log \left(\frac{p_{\text {eq }}^{\text {bound }}}{p_{\text {eq }}^{\text {bulk }}} \frac{V_{u}}{V_{0}}\right),
$$

where $k_{B}$ is the Boltzmann constant, $\mathrm{T}$ the temperature, $p_{e q}^{\text {bound }}$ the equilibrium probability of the bound state, $p_{e q}^{b u l k}$ the equilibrium probability of the bulk, $\mathrm{Vu}$ is the unbound volume and $\mathrm{V} 0=1661 \AA 3$ is the standard volume at 1 molar. The unbound volume can be measured in terms of the number of water molecules ( 55.55 waters in V0). Finally, the value for the $K_{e q}$ can be computed as follows.

$$
K_{e q}=e^{\Delta G / k_{B} T}
$$




\section{Figures}

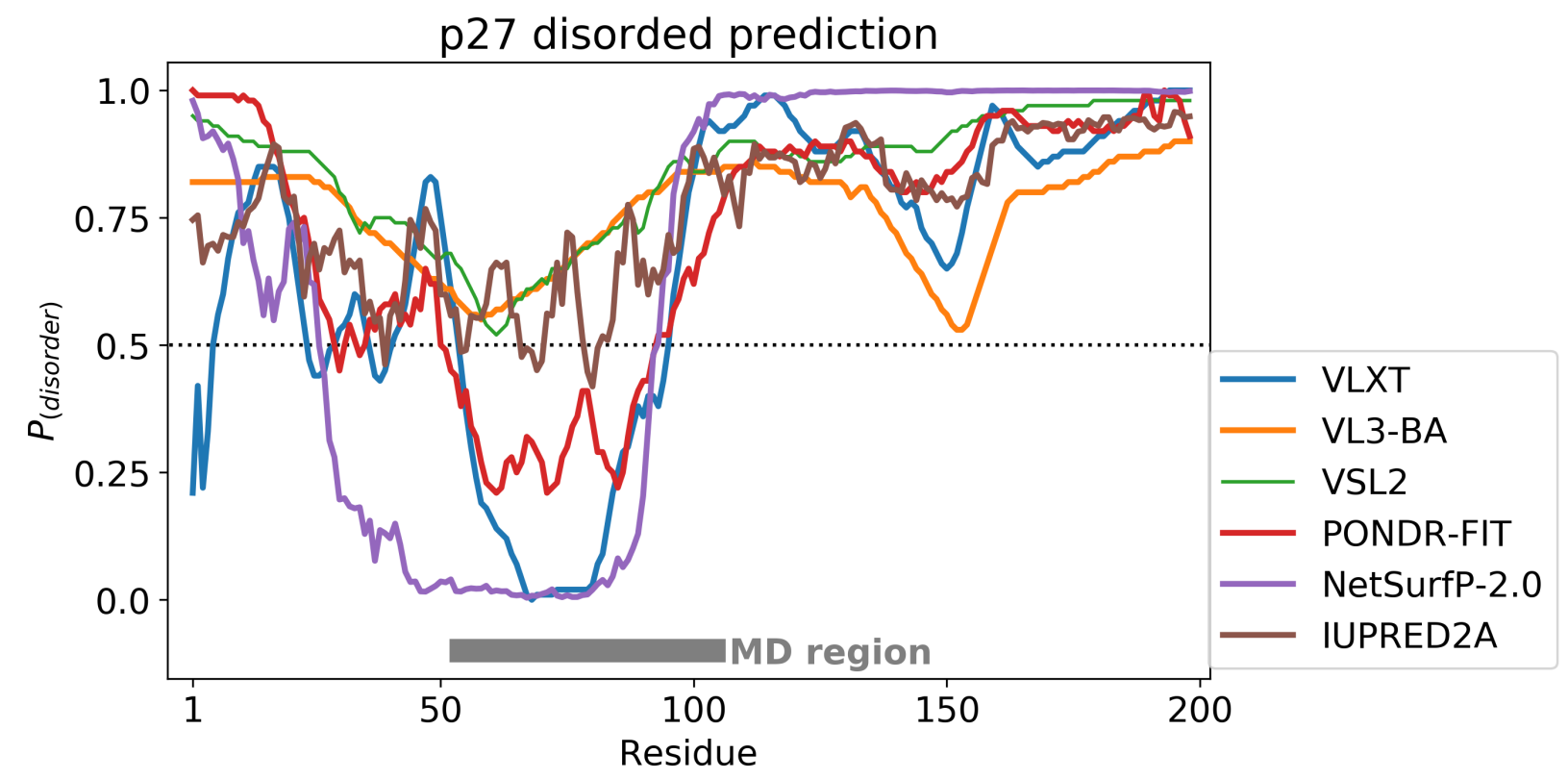

Figure 1: Disorder profile for p27. Several disorder prediction methods (VLXT, VSL2, VL3, ${ }^{1}$ PONDR-FIT, ${ }^{2}$ NetSurfP-2.0 ${ }^{3}$ and IUPRED2A ${ }^{4}$ ) were used. The section of protein used in the study spammed residues 54 to 101 (highlighted in grey). It corresponds to the region that folds as an $\alpha$-helix upon binding to the Cdk2-Cyclin A complex. ${ }^{5}$ 


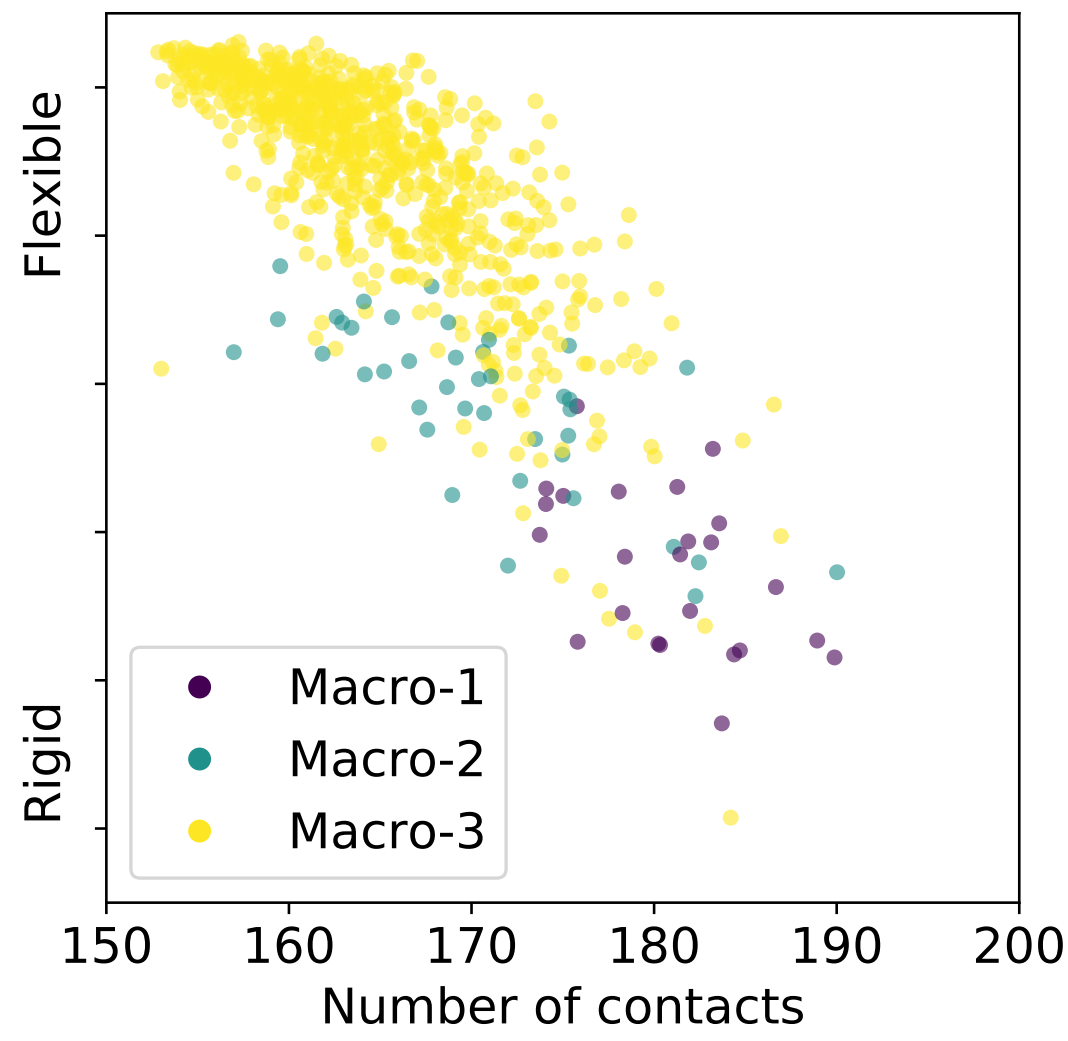

Figure 2: p27 MSM microstate distribution according to the number of contacts $x$-axis, flexibility $y$-axis measured as the sum of the deviation of backbone angles, and colored by macrostates assignment. 


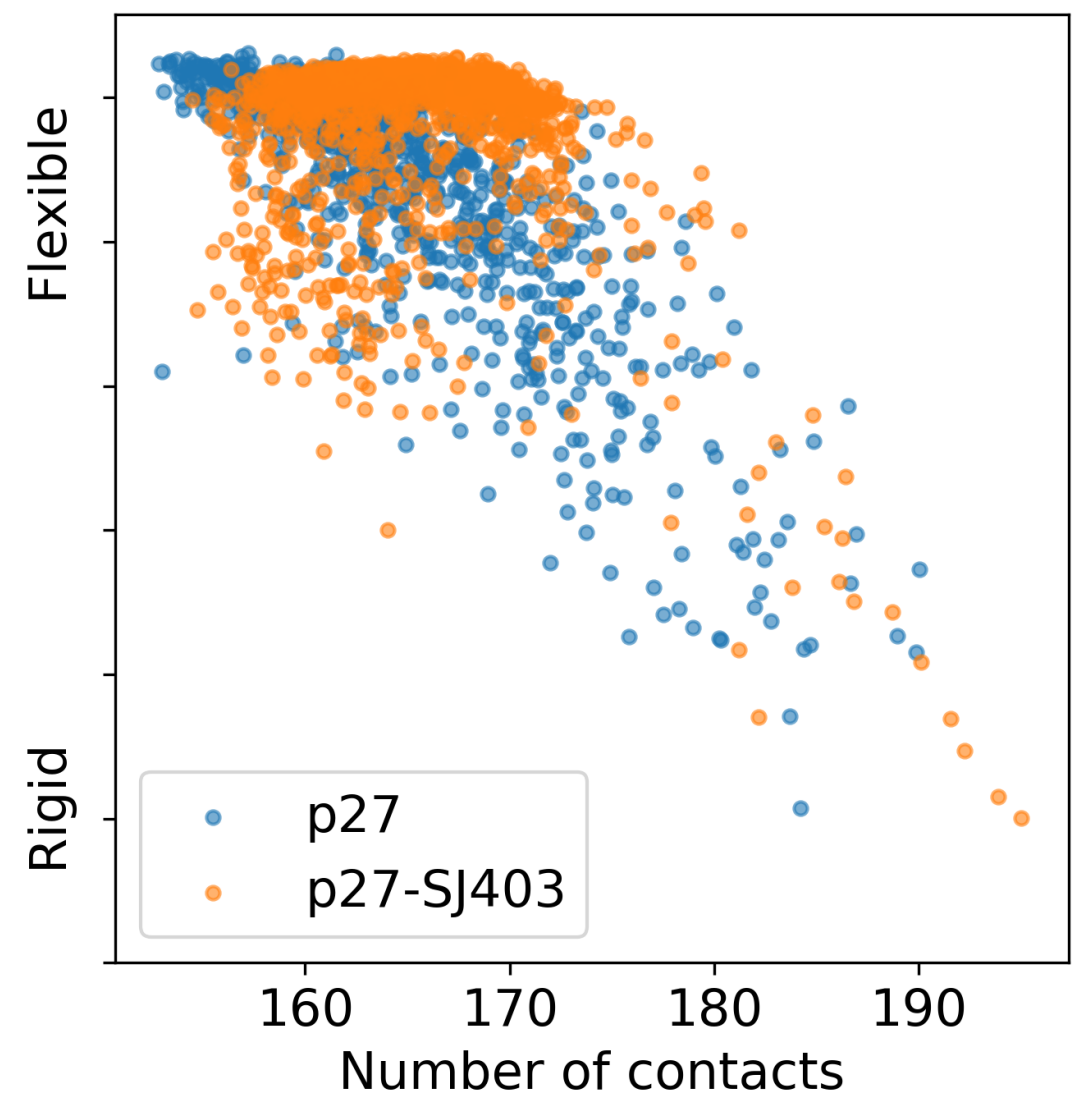

Figure 3: Comparison microstate distribution according to the number of contacts $x$ axis, flexibility $y$-axis measured as the sum of the deviation of backbone angles, and colored by macrostates assignment between the $p 27$ (isolated) and the p27-SJ403 data sets.
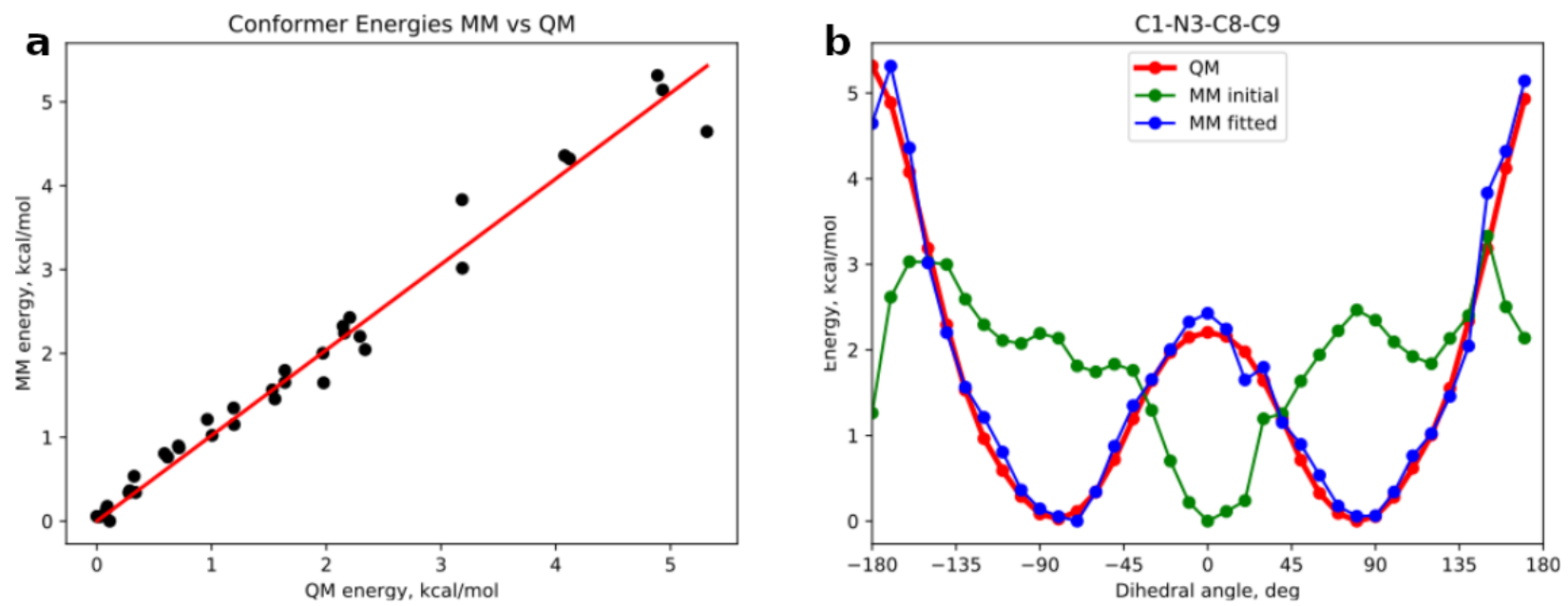

Figure 4: Molecule parametrization. a) Conformer energies. b) Fit of dihedral angles energy profile. Comparison of the quantum mechanics (in red) energy profile and the molecular mechanics before (in green) and after (in blue) being fitted. 

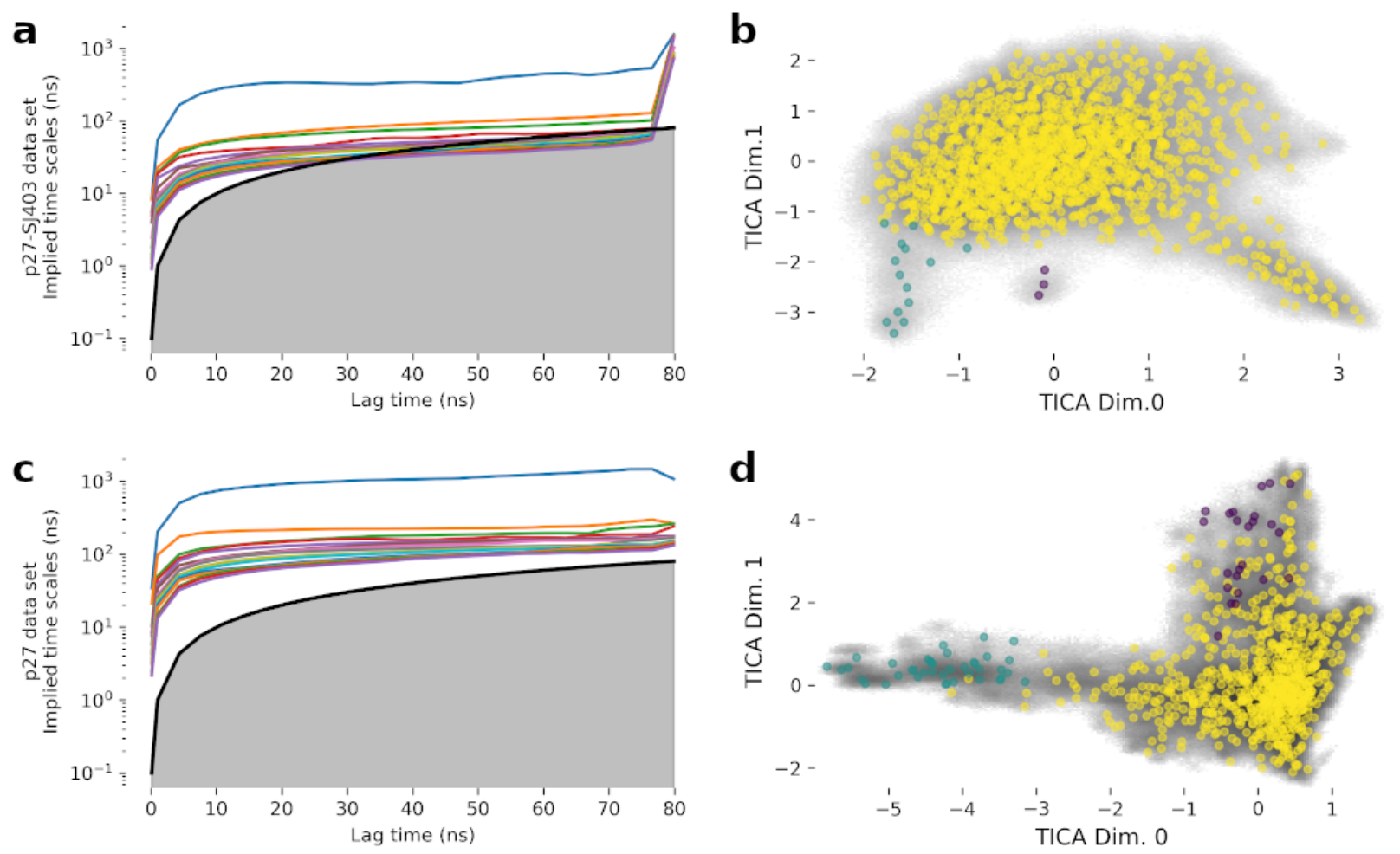

Figure 5: Models summary a) and c) Implied times scales for the p27SJ403 and the $p_{27}$ data set, respectively. b) and d) Microstates distribution over the first two TICA dimensions. 


\section{References}

(1) Obradovic, Z.; Peng, K.; Vucetic, S.; Radivojac, P.; Brown, C. J.; Dunker, A. K. Predicting Intrinsic Disorder From Amino Acid Sequence. Proteins: Struct., Funct., Bioinf. 2003, 53, 566-572.

(2) Xue, B.; Dunbrack, R. L.; Williams, R. W.; Dunker, A. K.; Uversky, V. N. PONDRFIT: a Meta-predictor of Intrinsically Disordered Amino Acids. Biochim. Biophys. Acta, Proteins Proteomics 2010, 1804, 996-1010.

(3) Klausen, M. S.; Jespersen, M. C.; Nielsen, H.; Jensen, K. K.; Jurtz, V. I.; Sønderby, C. K.; Sommer, M. O. A.; Winther, O.; Nielsen, M.; Petersen, B., et al. NetSurfP-2.0: Improved Prediction of Protein Structural Features by Integrated Deep Learning. Proteins: Struct., Funct., Bioinf. 2019, 87, 520-527.

(4) Mészáros, B.; Erdôs, G.; Dosztányi, Z. IUPred2A: Context-dependent Prediction of Protein Disorder as a Function of Redox State and Protein Binding. Nucleic Acids Res. 2018, 46, W329-W337.

(5) Russo, A. A.; Jeffrey, P. D.; Patten, A. K.; Massagué, J.; Pavletich, N. P. Crystal Structure of the p27Kip1 Cyclin-dependent-kinase Inhibitor Bound to the Cyclin ACdk2 Complex. Nature 1996, 382, 325-331. 